\title{
Productivity Challenges in Digital Transformation and its Implications for Workstream Collaboration Tools. *
}

\author{
Adela del-Río-Ortega \\ SCORE Lab, I3US Institute \\ Universidad de Sevilla \\ adeladelrio@us.es
}

\author{
Joaquín Peña \\ I3US Institute \\ Universidad de Sevilla \\ joaquinp@us.es
}

\author{
Manuel Resinas \\ SCORE Lab, I3US Institute \\ Universidad de Sevilla \\ resinas@us.es
}

\author{
Antonio Ruiz-Cortés \\ SCORE Lab, I3US Institute \\ Universidad de Sevilla \\ aruiz@us.es
}

\begin{abstract}
Digital transformation has brought an unprecedented pace of change and a huge amount of information available for businesses. At the same time, it has also created a number of difficulties for knowledge workers that have to deal with increasingly Volatile, Uncertain, Complex, and Ambiguous (VUCA) environments. In this scenario, the use of workstream collaboration tools (WSC), such as Microsoft Teams or Slack, to manage this new way of working and to improve the productivity of knowledge workers is proliferating. However, the goals that these WSC tools need to achieve and the way to use them are not well established because of two reasons: (i) these new work environments pose a new set of challenges for working productively that have not been clearly characterised, and (ii) there is neither previous experience nor a strong research body that study them in conjunction to offer guidelines to design good solutions based on WSC tools. In this paper, we follow an inductive approach based on the analysis of qualitative and quantitative data from 365 employees of 3 companies (immersed in VUCA environments and digitisation initiatives with WSC tools) to characterise the productivity challenges in these scenarios. The result is a set of 14 challenges that appear with different intensity in each company. A thorough study of the related literature shows that the implication of these challenges in WSC tools have been studied independently, but there is no single theory that covers all of them together. This paper, hence, helps to put them together.
\end{abstract}

\section{Introduction}

With the expansion of digital transformation [1], the amount of information and communication knowledge

This work has received funding from grants RTI2018-101204-B-C22 (OPHELIA) and RTI2018-101204-B-C21 (HORATIO) (MCI/AEI/FEDER, UE), and P18-FR-2895 (EKIPMENT+) from the Andalusian government and FEDER. workers have to manage has been growing exponentially in the last two decades. At the same time, the pace of work has sped up and the work has become more unstructured. In this situation, it is more common than ever to find organisations where a significant part of the daily work comes in the form of informal or unstructured processes, interruptions, or simply a set of unrelated tasks [2]. Recently, the term VUCA (Volatile, Uncertain, Complex and Ambiguous) has become popular to characterise this new way of working and its environment [3].

In this scenario, workstream collaboration (WSC) technology, being Slack and Microsoft Teams two of the most well-known of them are largely spreading among companies as a way to address the challenges posed by these new environments $[4,5]$.

WSC is a concept coined in 2018 that refers to "products that deliver a persistent conversational workspace for group collaboration and can be arranged into public or private channels (often organized by topic/project)" [6]. These tools are designed to improve team coordination, performance, communications, and productivity [4]. This emergent class of collaboration technology can combine a diverse number of features including instant messaging, calls, optimised search,, (shared) calendars and notifications, real-time document collaboration, task managers, and cloud storage with version control, amongst others. They also typically integrate with other enterprise applications and bots and can be accessed on mobile or desktop devices.

Besides, it is important to notice that the deployment of collaboration tools is not enough [7]. Instead, it is necessary a clear definition of the goals that WSC tools need to achieve, new ways of managing organisations, and change their culture so that the way to use these tools is focused on increasing knowledge workers' productivity and solving the challenges of digitised VUCA workplaces $[8,9]$.

This gains special importance in the context of WSC tools since they are very flexible and highly configurable and cannot be used as-is. To benefit from WSC, Users 
need to define how to use and configure them and also how they must be integrated with other applications according to each company's needs.

Unfortunately, these goals and ways of use are not well established yet because of two reasons: (i) these new work environments pose a new set of challenges for working productively that have not been clearly characterised and (ii) there is neither previous experience nor a strong research body that offer guidelines to design good solutions based on WSC tools considering all of those challenges together.

In this paper, we follow an inductive approach based on the analysis of qualitative and quantitative data from 365 employees of 3 companies, immersed in VUCA environments and digitisation initiatives with WSC tools, to characterise the productivity challenges in these new environments. This approach has two main differences in comparison with previous work. First, the challenges are obtained exclusively based on empirical evidence after analysing an open-ended question instead of using empirical evidence based on questionnaires to validate an existing theoretical model like in $[10,11]$ or not using empirical evidence at all [12]. Second, unlike previous work that cover all kinds of organisations, the analysis performed in this paper includes only companies that are immersed in VUCA environments and digital transformation efforts. This helps to provide a more precise image of the productivity challenges in these environments.

The result obtained from this analysis is twofold. On the one hand, we have identified a set of 14 challenges that can be used to define the goals that WSC tools need to achieve. On the other hand, we have found that although all challenges are present in the 3 organisations, they appear in each of them with different intensity. This suggests that the way to approach productivity improvements and the design of a solution based on WSC tools may change in intensity to adapt to these different productivity profiles of organisations. Finally, a thorough study of the related literature shows that the use of WSC tools to help address these challenges have been studied independently, but there is no single theory that covers all them together. This paper, hence, helps to put them together and can be used as a first step to build guidelines to use, configure and integrate WSC tools according to each company's needs.

The rest of the paper is organised as follows. Section 2 discusses related work. Section 3 describes the research methodology and threats to validity. The challenges identified are presented in Section 4, and based on them we analyse the differences between the studied companies in Section 5. The implications of the challenges detected for WSC tools are discussed in
Section 6. Finally, Section 7 concludes the paper and proposes some future research directions.

\section{Related Work}

There are two streams of work related to this paper, namely the research on factors that affect knowledge worker's productivity, and the research on how WSC tools can be used to help to address them.

Concerning the former, two broad groups can be identified. The first group focuses on identifying how a certain aspect impacts knowledge work productivity. Examples can be found for interruptions [13], email management [14] or multi-tasks [15], amongst others. Also, many techniques to improve knowledge work productivity have been proposed, each of them with a different focus, ranging from reducing stress [16] to increasing collaboration [17]. These studies are useful to understand how a factor influences productivity, but they do not allow a comparison of which factors have greater or lower influence.

This latter aspect is partially covered by the second group, which proposes models on which aspects affect productivity. Drucker identified in [18] 6 factors: work definition, self-management autonomy, innovation, continuous learning, quality over quantity, and motivation. In contrast, Davenport et al. [12] approached this topic from a more general perspective. According to them, three main factors drive knowledge work productivity: management and organisation, information technology, and workplace design. These three factors also appeared in Bosch-Sijtsema et al.'s framework [11], but it also includes teamwork processes, team structure, and team task. Other researchers consider some other drivers like mental space [19] or individual work practices [20].

Finally, Palvalin [10] is the first one that analyses knowledge work productivity in a general way, evaluating the significance of different drivers by conducting an empirical study based on the theoretical framework proposed. His conceptual model of knowledge work productivity consists of two major elements: work environment, which includes physical, virtual, and social environment, and knowledge worker, which includes individual work practices and well-being at work. The evaluation of the conceptual model in 9 companies confirms the positive relationship of the last three ones. Although closely related, this study differs from ours in several points. First, the survey used allowed the evaluation of a closed set of factors

The second stream of related work focuses on how WSC tools can be used to help to address factors that affect productivity. One line of work in this 
context has focused on understanding how WSC tools like Slack are used in different contexts like software engineering [21, 22], education, or libraries [23, 24, 25]. Others analyse the use of WSC tools for specific tasks like coordination [26, 27, 28] or knowledge management [29], or its impact on interruptions [30]. Finally, the third line of work explores how the use of bots and the integration capabilities of WSC tools can help to improve productivity $[31,32]$. However, all of these papers either focus on one specific productivity problem or some specific use of WSC tools, but do not provide a complete view that connects WSC tools with a model of knowledge worker productivity.

\section{Research Methodology}

The main reason for conducting this study was to characterise the productivity challenges in VUCA environments and identify how solutions based on WSC tools can help to address them. We were also interested in finding out whether these challenges were common to all organisations or there was a significant difference between them. This would help to know if it is possible to design solutions based on WSC tools that are useful for multiple organisations. This main objective was materialised in the following research questions:

RQ1. What are the main challenges that knowledge workers face in VUCA environments for being productive and managing working time?

RQ2. To what extent are these challenges repeated in different organisations?

RQ3. How can WSC tools help to address these challenges?

\subsection{Study Design}

We selected three big companies with international expansion from three very different sectors: Retail, commodities, and manufacturing ${ }^{1}$. These three companies were selected for several reasons: 1) their involvement in digital transformation initiatives to address the difficulties that their knowledge workers have to deal with increasingly VUCA environments; 2) their purpose to deploy a WSC tool to improve their knowledge worker productivity; 3) the different domains they belong to and 4) their willingness to participate in the study. A sample of 365 knowledge workers was involved in the study: 175 from the retail company, 86 from the commodities company, and 102 from the manufacturing one. These

\footnotetext{
${ }^{1}$ Their identities cannot be revealed for confidentiality reasons.
}

numbers correspond to a response rate of $98 \%$, a high but reasonable value given the interest of both the knowledge workers and their managers in identifying what hinders their productivity. All participants took part in the study voluntarily and all data were treated strictly confidentially after the data collection.

Regarding the data collection, we conducted an online survey consisting of 9 questions. The most important question, because it is the backbone of our study, was an open-ended question that provided qualitative data. Namely: "What are your main problems/improvement points when it comes to being productive and managing your time better?" The remaining questions were number questions, including percentages and semantic differentials, which provided quantitative data regarding different aspects: the time devoted to meetings (weekly) and interruptions (daily work time percentage); the number of emails received daily, the number of goals pursued simultaneously and the number of tasks in their ToDo list. All data collected was stored in spreadsheets to facilitate its analysis.

As for the data analysis, which is reported on in Sections 4 and 5, We first used an inductive approach consisting of coding the qualitative data into categories [33]. Then, we applied statistical techniques to identify the most frequent categories and to find correlations between them and the number of questions. Next, we used again several statistical techniques to analyse the differences amongst companies.

Finally, to identify how WSC tools can support knowledge workers to address these challenges, we conducted a thorough analysis of the related literature. Founded upon existing research, we described what WSC tools can do in each of the identified challenges and outlined some recommendations to improve WSC tools for this purpose (c.f. Section 6).

\subsection{Threats to Validity}

When it comes to the analysis of qualitative data, according to Padgett [34], most threats to validity fall within three broad headings: reactivity, researchers bias, and respondent bias. Reactivity refers to the potentially distorting effects the presence of the researcher may have on the studied setting, in particular the behaviour of the studied people [35]. Researcher bias refers to the preconceptions of the researcher, brought into the studied situation, which may impact on how the researcher asks questions or interprets answers. Respondent bias is based on the respondents' attitudes towards the study; it may be suspicion, leading to withholding information, or on the other end of the scale, companionship, leading to attempts to give the 
answer they think the researcher wants [36].

Regarding reactivity, we do not see any threat since the study we conducted was an online survey and the respondents were not in contact with the researchers either during the process of answering it or before.

Concerning researcher bias, the possible threats are mainly related to the way the data obtained was interpreted. To mitigate this threat, we used triangulation by involving three researchers with different backgrounds and levels of expertise in the context of knowledge worker productivity. They performed the analysis independently first and then a common discussion allowed for possible preconceptions of some of the researchers to be balanced by the others. We also believe that the three rounds of analyses performed (c.f. Section 4) helped to reduce this threat.

As for the respondent bias, this could be seen as the most important threat in our study. We start from the basis that our study is a subjective one since we analyse the ideas and perceptions expressed by respondents and not objective data. Nevertheless, a couple of countermeasures were applied. First, respondents were explicitly told the survey was anonymous (not shared with their company) and was aimed to help them to solve their problems. Second, as a countermeasure for the respondent bias in the open-ended question, we added a battery of number questions that force respondents to further analyse and numerically estimate certain aspects that can be related to their productivity, such as the number of meetings they have weekly or the time they spend daily managing email. In this way, we can identify if there is a correlation between the problems described in the answers provided to the open-end question and the information obtained from the number of questions.

Another important aspect to be taken into account is the external validity of our study, which describes the possibility of generalising its results. In this research, 365 practitioners of 3 companies from three very different sectors were surveyed. In addition, there was no previous relationship between the researchers and the participants, and there was no further interest by the respondents to participate in the survey than finding hints to improve their productivity. Of course, there can be specific sectors or companies where our findings do not apply, but in general, we believe the results obtained in this study can be generalised to knowledge workers of any company that meets the characteristics of the ones under study, i.e. big companies that are immersed in the digital era experimenting changes in the way work is approached and performed.

\section{Productivity Challenges}

To answer RQ1, we applied an inductive approach to analyse our qualitative data by coding it into categories. We followed the procedure described in [33] for this purpose. It was performed by three different researchers and included three rounds. First, each of the three researchers read closely the answers of participants, identified categories, and refined them. After this, the three researchers met to pool the categories obtained. In this first round, the number of categories ranged from 15 to 26 amongst the three researchers, and we agreed in the meeting to establish 16 categories and to reclassify the data according to those categories. A second meeting was required to discuss the differences in the classification and to clarify the meaning of some categories that had been differently interpreted by the researchers. Finally, a subset of 14 categories was established as the definitive set of categories to be considered for the study. Their description is as follows.

Interruptions: It comprises problems related to a high frequency of occasions when someone or something stops you from working on your duties. They include calls (personal or professional), emergencies, unplanned/unexpected events or requests from others.

Meetings: It comprises problems related to meetings such as being too long, or to frequent, or unproductive.

Email management: It comprises problems related to the organisation of email, including reading, processing, answering, and forwarding.

Software: It comprises all references to problems related to software, either due to its inefficiency, ineffectiveness or non-existence.

Planning/task management: It refers to problems related to the lack of organisation of tasks along a time period as well as milestones with associated deadlines. It also includes problems related to not following the plan.

Prioritisation/goals: This category encompasses all references to problems related to deciding which of a group of things/tasks are the most important to deal with them first, i.e., they are more aligned with their goals. Therefore, this category also includes all problems related to the definition of goals, their order, conflicts between them, etc.

Volatility: It includes references to problems derived from sudden changes in some aspects relevant to 
work. It includes references to policy changes, priority/goals changes, a changing environment, differences in the workloads depending on seasons or other circumstances.

Bureaucracy: This category includes problems related to complicated rules, processes, reporting, and written work that make it hard to get tasks done. There are many references to inefficient processes and administrative burdens.

Work overload: It includes references to too many tasks, projects, not enough time, or lack of personnel.

Information overload: This category refers to the existence of too many information sources, channels, or platforms, as well as non-structured information or difficult to organise. Some respondents use the recently popular term infoxication to refer to this situation.

Organisational coordination: It comprises references related to problems with other departments, colleagues, or managers. It includes coordination, delegation, and follow-up problems as well as poor communication that prevents the information from reaching the right people at the right time.

Motivation: It refers to problems related to the lack of enthusiasm for working. It also includes mentions of lack of future prospects or recognition (usually from superiors).

\section{Lack of knowledge or training: It}

comprises references to unqualified or inexperienced (or at least not qualified or experienced enough in certain areas) personnel, either the participant herself or her subordinates/colleagues.

Concentration/focus: It comprises problems related to a limited ability to think carefully about the current task and nothing else, including lack of concentration due to a noisy environment.

Once established these categories, a final and common classification was performed by the three researchers together. We established the criteria for selecting one category per sentence/block of text related to the same idea in each participant answer. In those cases when different categories could be associated with one sentence, only the most prominent category was selected. The output of this final classification is the set of productivity challenges.

Looking at the frequencies in which the challenges are mentioned by the participants (cf. Table 1),

\begin{tabular}{|l|c|}
\hline Category & Freq. \\
\hline Interruptions & 121 \\
Prioritisation/goals & 88 \\
Organisational coordination & 67 \\
Planning/task management & 57 \\
Work overload & 34 \\
Lack of knowledge or training & 25 \\
Email management & 21 \\
Volatility & 21 \\
Concentration/focus & 17 \\
Bureaucracy & 17 \\
Meetings & 15 \\
Software & 14 \\
Motivation & 13 \\
Information overload & 12 \\
\hline
\end{tabular}

Table 1. Frequencies in which participants mention each category

we conclude that the most frequent problem is by far Interruptions. Then, there are three problems: Prioritisation/goals, Organisational coordination, and Planning/task management, which are also rather frequent. A third group includes the remaining categories, in which the less frequent ones are Information overload, Motivation, and Software.

Finally, we applied a Kruskal-Wallis test between the quantitative questions of our survey and the categories, obtaining the following results: Time for emails is highly related to Email management $(p<0.01)$; Time for meetings is related to Meetings, Work overload, and Prioritisation/goals $(p<0.01)$; Percentage of interruptions is highly related to Interruptions, Information overload, Meetings, and Prioritisation/goals $(p<0.05)$; Stress is highly related to Organisational coordination, Work overload and Prioritisation/goals $(p<0.05)$; Number of tasks is highly related to Prioritisation/goals and Work overload $(p<0.05)$, and Number of goals is highly related to Bureaucracy, Email management, Organisational coordination, and Planning/task management $(p<0.05)$

Many of these relations are what one could naturally expect, e.g., the relationship between time for emails, time for meetings and percentage of interruptions, and their corresponding categories (Email management, Meetings, and Interruptions) or the relationship between stress and work overload. This gives us greater confidence in the results of the qualitative analysis performed. Other relationships present in the data, such as between time for meetings and work overload or prioritisation/goals, are also expected because many of these challenges are interrelated to each other. For instance, a bad goal definition may require additional 
meetings to clarify the objectives.

\section{Analysis of Differences between Organisations}

In this section, we aim at pinpointing the differences regarding the challenges between the three organisations under study (RQ2). If we look at the frequencies of the categories grouped by the organisation of the participant that mentions them (cf. Figure 1) we can observe that all challenges were found in all organisations. This suggests that they are rather generic and not specific to an organisation or sector. Another observation is that some challenges (e.g. interruptions or prioritisation/goals) are much more frequent in all organisations than others (e.g. bureaucracy or information overload). This may suggest that there are challenges that are more prominent in the VUCA environment in which all companies analysed are immersed. The fact that the challenges typically identified in VUCA context are those that are more prominent in all organisations supports this idea. It also gives a good starting point for future studies focused on looking into the differences in productivity challenges between VUCA and non-VUCA environments.

Figure 1 also shows differences between the intensity in which each challenge appears in each organisation. In fact, an analysis of the factors that influence the organisation using a Chi-square test of independence of variables shows that the differences between the organisations in Motivation, Interruptions, Email management, and Software are statistically significant $(p<0.05)$, and the first two in particular are very significant $(p<0.01)$. One can think of two possible reasons for these differences, namely external reasons or internal reasons. The first one refers to the type of work performed by the organisation or the external context in which this work is performed. For instance, organisations in some specific domain like retail could be more prone to interruptions than others in a manufacturing domain. The second one refers to factors that are internal to the organisation such as the management techniques used or the abilities or knowledge of their staff.

Regardless of the reason, the difference is relevant because it means that we are able to identify challenges that seem more prominent in an organisation than other. Therefore, the 14 challenges identified in this paper can be a useful tool to build productivity profiles that can be used to benchmark organisations in terms of their challenges for being more productive.

\section{Implications for WSC Tools}

The 14 challenges identified have implications on the requirements WSC tools have to fulfill. Next, we outline these implications by detailing how WSC tools can help to address those challenges (RQ3). Specifically, following a deductive approach, we perform an analysis of existing research to report on what WSC tools can do with regard to each of these challenges (or set of related challenges) and outline some recommendations to improve WSC tools for that purpose. This work can help to build guidelines to use, configure and integrate WSC tools according to each company's needs.

Interruptions and Concentration/Focus: WSC tools offer instant messaging features that can benefit workplace communication: the ease of coordination and scheduling; its immediacy, with nearly-synchronous communication; its informal nature makes it a very useful tool [37]. However, they often result in interruptions that preempts the knowledge worker from his primary task [30]. The importance for knowledge workers to maintain a state of "flow" has been broadly reported [38] and interruptions disrupt this state of flow. A system automatically collecting and sharing information about the availability of colleagues could allow a person to use that information to decide whether it is appropriate to initiate communication [39]. Following this idea, different computer-based mechanisms can be used to reduce interruptions and distractions. Bots, integrated into the WSC tool used, can be aware of the workers context and defer interruptions and notifications until a more suitable time [38]. Speech detection sensor data, location information, computer activity, and calendar information can be combined to model availability for communication [40]. These mechanisms might be further complemented with others that deal with in-person interruptions, like in [41] where a physical LED light indicates if the user can be interrupted automatically using a computer application. Finally, for those interruptions that are unavoidable, bots integrated into WSC tools like Slack could also support context switching by integrating tools reducing friction from tool switching, or by gathering information that was formerly scattered across various tools [31].

\section{Prioritisation/objectives, Planning/Task Management, Organisational Coordination,} Volatility, and Motivation: Setting goals and prioritising them in VUCA environments require a new management and leadership style. The flexibility and adaptation to change of "Agility", agile planning 


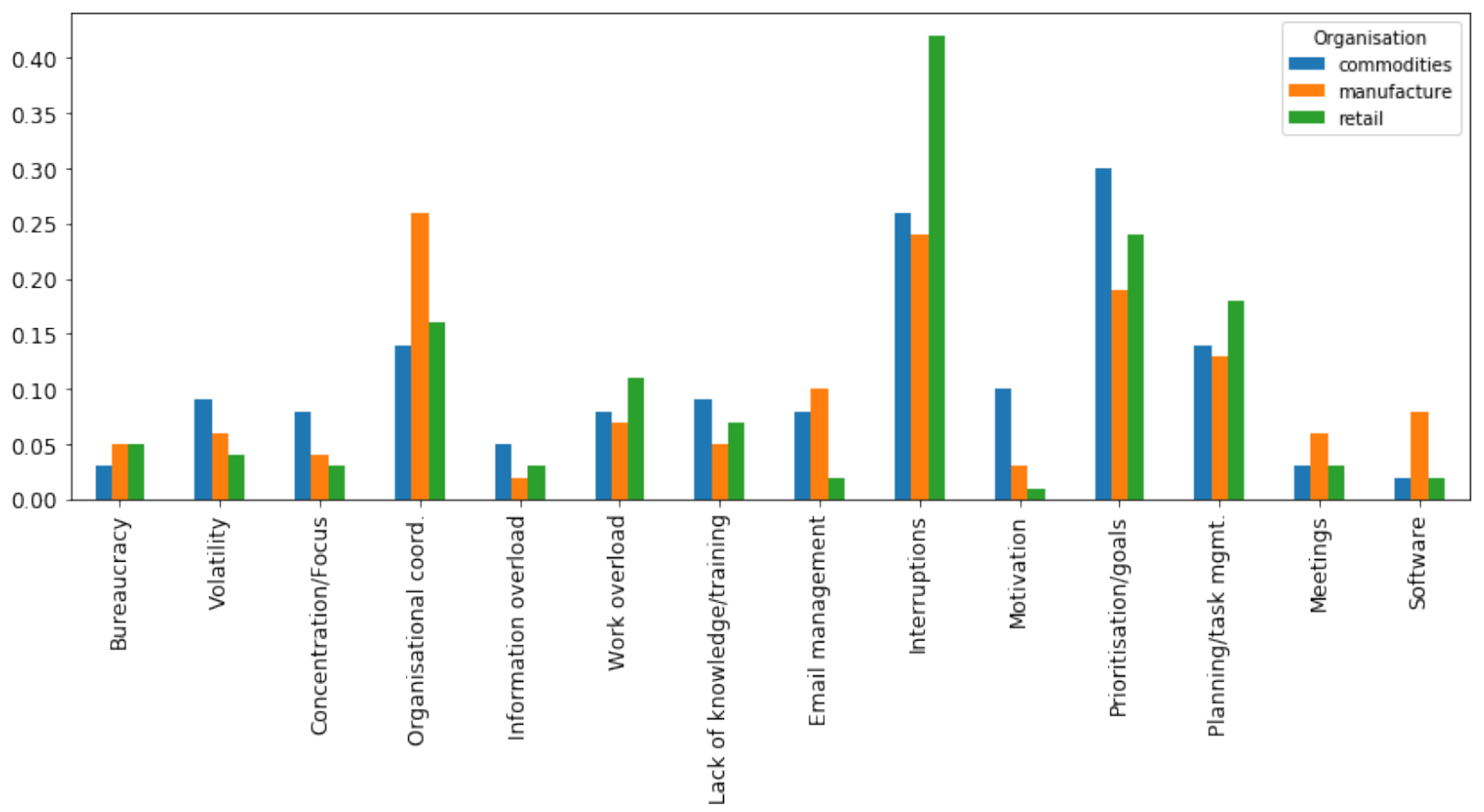

Figure 1. Frequency of productivity challenges grouped by organisation

methods and organisational structure have been presented as imperative for VUCA environments [9]. These methods maintain the needed flexibility but impose order and communication paths that can reduce interruptions, improve coordination, set clear priorities (sprints), and order/plan the task to be performed [42].

In rapidly changing environments, a management style based on control and traditional planning does not work and provokes the identified challenges; in contrast, a more "distant leadership" and culture is needed for giving flexibility and delegating decisions and prioritisation to team members [8].

In this context, the use of collaboration tools is presented as convenient by many authors for three main reasons. 1) They allow a new management style needed in VUCA digitised environments: Collaboration tools for knowledge sharing (i.e. Slack or Teams) and task sharing (i.e. Trello or Planner) are needed to enable this new way of collaboration in a knowledge-sharing-based and community-oriented-based model where work is structured in "convergent interactions", in contrast with the old "divergent models" driven by email [27, 28, 8]. 2) They increase motivation: WSC tools are presented as needed for the implementation of agile planning and "distant leadership" models and have proved to increase the autonomy of team members and employee motivation [8]. 3) They increase productivity: WSC tools have been studied in supply chains (several companies coordinating to produce goods to the end customer) concluding that its use, particularly regarding coordinated planning, increases the performance of participants and of the global chain [43], and similar results are found in non-supply chains companies [8].

Thus, as shown above, WSC tools should provide specific mechanisms to manage agile culture, agile planning, knowledge sharing, and collaborative task management. In addition, mechanisms for the measurement and analysis of goals, plans, KPIs, and people's performance have been presented as convenient $[44,45]$.

Bureaucracy: Rigid and obsolete procedures are one of the main problems related to bureaucracy. Some works exist in the context of electronic resource management [23, 25] or in the context of project management $[24,46]$ that report on the use of tools like Trello, integrated with Slack, or Planner, integrated with MS Teams, as workflow management tools that combined with agile methods provide ways to save time and handle work more efficiently.

Meetings: Virtual meetings, i.e. audio-, video- and web-conferencing, can help reduce the productivity of employees if supplemented with other virtual collaboration tools [47]. Furthermore, the importance of collaboration tools to support an effective coordination 
structure, with both scheduled and unscheduled meetings, is highlighted in [26]. It reports on the benefits of using Slack for this purpose in the context of global software engineering but stresses the need to define procedures, related also to the aforementioned mechanisms to avoid interruptions, for the use of such tools so that everyone can benefit from them. Finally, monitoring the time spent in meetings can provide valuable information to analyse whether a reduction of meetings and their duration is necessary [48].

Email Management: WSC tools have been reportedly used to successfully replace email for many communication needs in an organisation $[29,22]$ because they provide several topic-based communication channels instead of one single inbox, which facilitates knowledge management [29]. However, as shown in [49], habit plays an important role in the success of changing email with social-collaboration technologies, which means that continuous change management efforts are necessary to ensure the WSC tools are widely used. Furthermore, WSC tools must provide mechanisms to monitor its usage and capture how efficiently knowledge is created, shared, and retrieved across the organisation [49]. Finally, email exchanges with external collaborators will still be needed, so mechanisms to connect email with WSC tools should be provided.

Information Overload: The ability of WSC tools to aggregate information from multiple sources and to organise information in several channels has been proposed as a way to deal with information fragmentation and overload in fields like software development, where the WSC tool collects information from several continuous integration and continuous deployment tools [32]. In fact, these features make the WSC tool work like a dashboard, which is commonly used as a way to alleviate information overload $[50,51]$. Furthermore, [29] has shown how these features on Slack can lead to effective identification and structuring of knowledge areas and resources, which helps to decrease information overload. However, current WSC tools are limited to just show an aggregated stream of information and filtering has to be done explicitly by searching. Future WSC can use AI techniques to filter results based on the current context and improve situational awareness as shown in $[52,53]$.

Lack of knowledge or training: WSC tools can help to address this challenge from different perspectives. On the one hand, they are structured in a way that captures the team knowledge, namely, they register automatically the activity of a team/group organised by topic so that it is easy to search the social stream to learn how problems are being solved or the group usually works. This makes these tools well-suited for knowledge management, including transfer, organisation, and saving of knowledge as reported by [29]. On the other hand, WSC tools can also be used to facilitate learning. For instance, [22] reports on the use of Slack to participate in communities of practice and keep up with new tools and frameworks, and [54] describes how Slack was very useful for onboarding in distributed software teams because of its fast feedback and communication, which strengthens the level of trust. Finally, the analysis of WSC tools logs has also proven to be a valuable tool to understand the collaboration and communication going on in the organisation during the onboarding process [54], although few recommendations exist on how to analyze them.

Work overload: Implementing strategies to address work overload is challenging. According to [48], at the very least actions such as regular monitoring of workloads to enable an evaluation of the extent to which this is a significant issue and ensuring that employees are able to take sufficient breaks should be taken to avoid this issue. In this context, the analysis of WSC tools logs can again be useful to monitor the amount of work performed in real-time by measuring the time spent on each tool/project. Furthermore, sentiment analysis and natural language processing techniques could be applied to the logs like in [21]. This could help to detect potential problems derived from work overload. Finally, tools like Microsoft Analytics already provide some of these measurements and proactively suggests actions to ensure employees are taking sufficient breaks.

\section{Conclusions and Future Work}

WSC tools have emerged as a way to help knowledge workers manage the difficulties that have appeared within increasingly VUCA environments. However, the goals that these WSC tools need to achieve and the way to use them are not well established because these new work environments pose a new set of challenges for working productively that have not been clearly characterised and there is neither previous experience nor a strong research body that offer guidelines to design good solutions based on WSC tools.

In this paper, we have addressed the first issue by conducting an empirical study that involved 365 employees from 3 different companies of different sectors immersed in VUCA environments and digitisation initiatives. The main result is the 
identification of 14 productivity challenges and an analysis of the extent these challenges are repeated in different organisations. Furthermore, we have conducted a thorough study of the literature related to identifying reported ways in which WSC tools can help address these challenges.

There are two main conclusions from these results. First, there is a set of 14 productivity challenges that are present in all organisations which must be addressed by WSC tools. Furthermore, the fact that the four challenges with more frequency, i.e. interruptions, prioritisation/goals, organisational coordination, and planning/task management, are clearly aligned with the main goals that industry tries to achieve with WSC tools supports this idea and $[4,5]$ supports the validity of the study from a research and industrial point of view. It is also worth mentioning the differences in the intensity in which these challenges appear in each organisation. They suggest that it is possible to create productivity profiles of organisations based on the 14 challenges.

Second, the literature analysis reveals that the support of WSC tools for the 14 challenges have been studied independently in the literature, but there is no single theory that covers all of them together. In this paper, we have put them together describing what WSC tools can do in each of these challenges and outlining some recommendations to improve WSC tools for this purpose.

As for future work, several research lines are envisioned. First, we plan to carry out this study with more companies and participants to consolidate the results obtained. Particularly, it could be interesting to replicate the results with several companies in the same sector to analyse which part of the differences between organisations are caused by the different sector in which they are. It would also be interesting to extend the study to make a comparison between VUCA and non-VUCA challenges. As it is, the dataset used in our study does not allow us to establish what is special or unique to VUCA environments in the identified challenges. Finally, we would like to identify usage patterns of WSC tools and relate them with the productivity challenges identified in this paper so that we can design specific guidelines for solutions based on WSC tools.

\section{References}

[1] C. Matt, T. Hess, and A. Benlian, "Digital transformation strategies," Business \& Information Systems Engineering, vol. 57, no. 5, pp. 339-343, 2015.

[2] T. H. Davenport, "Process management for knowledge work," in Handbook on Business Process Management 1. Int. Handbooks on Inf. Sys., pp. 17-35, Springer, 2015.

[3] N. Bennett and J. Lemoine, "What vuca really means for you," Harvard Business Review, vol. 92, no. 1/2, 2014.
[4] Z. Kerravala and D. Michels, "Business agility drives the need for workstream communications and collaboration," ZK Research, August 2015.

[5] M. Gotta, A. Preset, and B. Elliot, "Embrace workstream collaboration to transform team coordination and performance," Gartner Research, May 2017.

[6] M. Gotta, G. Dewnarain, and A. Preset, "Market guide for workstream collaboration," Gartner Research, September 2018.

[7] R. Dawson, J. Hough, J. Hill, B. Winterford, and D. Alexandrov, Implementing Enterprise 2.0. Advanced Human Technologies Sydney, 2009.

[8] R. Busse and G. Weidner, "A qualitative investigation on combined effects of distant leadership, organisational agility and digital collaboration on perceived employee engagement," Leadership Org. Dev. J., 2020.

[9] S. Bill Pasmore PhD, "Leadership agility: A business imperative for a vuca world," People and Strategy, vol. 33, no. 4, p. 32, 2010.

[10] M. Palvalin, "What matters for knowledge work productivity?," Employee Relations, vol. 41, no. 1, pp. 209-227, 2019.

[11] P. Bosch-Sijtsema, R. Fruchter, M. Vartiainen, and V. Ruohomäki, "A framework to analyze knowledge work in distributed teams," Group and Organization Management, vol. 36, no. 3, pp. 275-307, 2011.

[12] T. Davenport, R. Thomas, and S. Cantrell, "The mysterious art and science of knowledge-worker performance," MIT Sloan Management Review, vol. 44, no. 1, pp. 23-30, 2002.

[13] P. Kalgotra, R. Sharda, and R. McHaney, "Understanding the impact of interruptions on knowledge work: An exploratory neuroimaging study," in Proc. of the Annual Hawaii Int. Conf. on Syst. Sciences, pp. 658-667, 2016.

[14] A. Gupta, R. Sharda, and R. Greve, "You've got email! does it really matter to process emails now or later?," Inform. Syst. Front., vol. 13, no. 5, pp. 637-653, 2011.

[15] S. Appelbaum, A. Marchionni, and A. Fernandez, "The multi-tasking paradox: Perceptions, problems and strategies," Management Decision, vol. 46, no. 9, pp. 1313-1325, 2008.

[16] C. Ipsen and P. Jensen, "Organizational options for preventing work-related stress in knowledge work," Int. J. Ind. Ergon., vol. 42, no. 4, pp. 325-334, 2012.

[17] G. Schuh, T. Potente, C. Wesch-Potente, and A. Weber, "Collaboration mechanisms to increase productivity in the context of industrie 4.0," Procedia CIRP, vol. 19, pp. 51-56, 2014.

[18] P. F. Drucker, "Knowledge-worker productivity: The biggest challenge," California management review, vol. 41, no. 2, pp. 79-94, 1999.

[19] V. M., "Analysis of multilocational and mobile knowledge workers' work spaces," in Engineering Psychology and Cognitive Ergonomics (H. D., ed.), pp. 194-203, 2007.

[20] J. Ruostela and A. Lönnqvist, "Exploring more productive ways of working," Int. J. of Social, Behavioral, Educational, Economic, Business and Industrial Engineering, vol. 7, no. 1, pp. 153-161, 2013.

[21] V. Stray, N. B. Moe, and M. Noroozi, "Slack me if you can! using enterprise social networking tools in virtual agile teams," in ACM/IEEE 14th Int. Conf. on Global Software Engineering (ICGSE), pp. 111-121, 2019. 
[22] B. Lin, A. Zagalsky, M.-A. Storey, and A. Serebrenik, "Why developers are slacking off: Understanding how software teams use slack," in Proc. of the 19th ACM Conf. on Computer Supported Cooperative Work and Social Computing Companion, pp. 333-336, Feb. 2016.

[23] K. Ostergaard, "Applying kanban principles to electronic resource acquisitions with trello," Journal of Electronic Resources Librarianship, vol. 28, no. 1, pp. 48-52, 2016.

[24] N. S. Jyothi and A. Parkavi, "A study on task management system," in Int. Conf. on Research Advances in Integrated Navigation Systems, pp. 141-147, 2016.

[25] E. M. Gould, "Workflow management tools for electronic resources management," Serials Review, vol. 44, no. 1, pp. 71-74, 2018.

[26] V. Stray and N. B. Moe, "Understanding coordination in global software engineering: A mixed-methods study on the use of meetings and slack," J. Syst. Softw., vol. 170, p. 110717, Dec. 2020.

[27] D. Chasanidou, B. Elvesaeter, and A.-J. Berre, "Enabling team collaboration with task management tools," in Proc. of the 12th Int. Symp. on Open Collab., pp. 1-9, 2016.

[28] M. Bettoni, W. Bernhard, N. Bittel, and V. Mirata, "The art of new collaboration: Three secrets," in Proc. of the 19th Europ. Conf. on Knowledge Management ECKM 2018, pp. 1133-1141, 072018.

[29] S. Dennerlein, R. Gutounig, E. Goldgruber, and S. Schweiger, "Web 2.0 messaging tools for knowledge management? exploring the potentials of slack," in Proc. of the 17th Europ. Conf. on Knowledge Management ECKM 2016, p. 225, 2016.

[30] A. Gupta, H. Li, and R. Sharda, "Should I send this message? understanding the impact of interruptions, social hierarchy and perceived task complexity on user performance and perceived workload," Decis. Support Syst., vol. 55, pp. 135-145, Apr. 2013.

[31] M.-A. Storey and A. Zagalsky, "Disrupting developer productivity one bot at a time," in Proc. of the 201624 th ACM SIGSOFT Int. Symp. on Foundations of Software Engineering, FSE 2016, pp. 928-931, Nov. 2016.

[32] F. Calefato and F. Lanubile, "A Hub-and-Spoke model for tool integration in distributed development," in 2016 IEEE 11th Int. Conf. on Global Software Engineering (ICGSE), pp. 129-133, Aug. 2016.

[33] D. R. Thomas, "A general inductive approach for analyzing qualitative evaluation data," American Journal of Evaluation, vol. 27, pp. 237-246, June 2006.

[34] D. K. Padgett, Qualitative Methods in Social Work Research. SAGE Publications, Inc., 2008.

[35] C. S. Franklin, P. A. Cody, and M. Ballan, "Reliability and validity in qualitative research," in The Handbook of Social Work Research Methods (B. Thyer, ed.), ch. 19, pp. 355-374, SAGE Publications, Inc., 2010.

[36] D. Karlström and P. Runeson, "Integrating agile software development into stage-gate managed product development," Empir. Soft. Eng., vol. 11, pp. 203-225, 2006.

[37] N. S. Baron, "See you online: Gender issues in college student use of instant messaging," J. Lang. Soc. Psychol., vol. 23, no. 4, pp. 397-423, 2004.

[38] M. P. Acharya, C. Parnin, N. A. Kraft, A. Dagnino, and X. Qu, "Code drones," in Proc. of the 38th Int. Conf. on Software Engineering Companion, pp. 785-788, 2016.
[39] T. Erickson and W. A. Kellogg, "Social translucence: An approach to designing systems that support social processes," ACM Trans. Comput.-Hum. Interact., vol. 7 , p. 5983, Mar. 2000

[40] J. Fogarty, J. Lai, and J. Christensen, "Presence versus availability: the design and evaluation of a context-aware communication client," Int. J. Hum. Comput. Stud., vol. 61, no. 3, pp. $299-317,2004$.

[41] Züger, M. et al., "Reducing interruptions at work: A large-scale field study of flowlight," in Proc. of the 2017 CHI Conf. on Human Factors in Computing Systems, pp. 61-72, ACM, 2017.

[42] S. Denning, "The age of agile," Strategy —\& Leadership, vol. 45, no. 1, pp. 3-10, 2017.

[43] O. R. Ovalle and A. C. Marquez, "The effectiveness of using e-collaboration tools in the supply chain: an assessment study with system dynamics," J. Purch. Supply Manag., vol. 9, no. 4, pp. 151-163, 2003.

[44] B. Estrada-Torres, P. H. Piccoli Richetti, A. Del-Río-Ortega, F. Araujo Baião, M. Resinas, F. M. Santoro, and A. Ruiz-Cortés, "Measuring performance in knowledge- intensive processes," ACM Trans. Internet Technol, vol. 19, no. 1, Article 15, pp. 1-26, 2019.

[45] I. Pappas, P. Mikalef, and M. e. a. Giannakos, "Big data and business analytics ecosystems: paving the way towards digital transformation and sustainable societies,' Inf Syst E-Bus Manage, vol. 16, p. 479491, 2018.

[46] N. Naik and P. Jenkins, "A web based method for managing prince2 projects using trello," in 2019 Int. Symp. on Systems Engineering (ISSE), pp. 1-3, 2019.

[47] P. A. Lindeblad, Y. Voytenko, O. Mont, and P. Arnfalk, "Organisational effects of virtual meetings," Journal of Cleaner Production, vol. 123, pp. 113 - 123, 2016.

[48] N. Skinner and B. Pocock, "Work-life conflict: Is work time or work overload more important?," Asia Pac. J. of Human Resources, vol. 46, pp. 303-315, Dec. 2008.

[49] J.-C. Pillet and K. D. A. Carillo, "Email-free collaboration: An exploratory study on the formation of new work habits among knowledge workers," Int. J. Inf. Manage., vol. 36, pp. 113-125, Feb. 2016.

[50] P. Gupta and A. W. Woolley, "Productivity in an era of multi-teaming: The role of information dashboards and shared cognition in team performance," Proc. ACM Hum.-Comput. Interact., vol. 2, Nov. 2018.

[51] D. Saxena and M. Lamest, "Information overload and coping strategies in the big data context: Evidence from the hospitality sector," J. Inf. Sci. Eng., vol. 44, pp. 287-297, June 2018.

[52] D. Raymond, "Using artificial intelligence to combat information overload in research," IEEE Pulse, vol. 10, pp. 18-21, Jan. 2019.

[53] E. Simperl, I. Thurlow, P. Warren, F. Dengler, J. Davies, M. Grobelnik, D. Mladenić, J. M. Gómez-Pérez, and C. R. Moreno, "Overcoming information overload in the enterprise: The active approach," IEEE Internet Comput., vol. 14, pp. 39-46, Nov. 2010.

[54] N. B. Moe, V. Stray, and M. R. Goplen, "Studying onboarding in distributed software teams: A case study and guidelines," in Proc. of the Evaluation and Assessment in Software Engineering, EASE '20, pp. 150-159, Apr. 2020. 